\title{
Dynamism in Resistance Pattern of Escherichia coli-A Drift from Indian Council of Medical Research (ICMR)-Antimicrobial Use Guidelines
}

\author{
V. D. Sathish ${ }^{1^{*}}$, Guru Prasad Mohanta ${ }^{2}$, \\ Elizabeth Anna Mathew ${ }^{2}$ and J. Renga Ramanujam ${ }^{3}$ \\ ${ }^{1}$ Department of Pharmacy Practice, KMCH College of Pharmacy, \\ Coimbatore, TamilNadu, India \\ ${ }^{2}$ Department of Pharmacy, Annamalai University, Chidambaram, TamilNadu, India \\ ${ }^{3}$ Department of Microbiology, DR.N.G.P Arts and Science College, Coimbatore, \\ Tamil Nadu, India \\ *Corresponding author
}

\section{A B S T R A C T}

Combating Antibiotic resistance has become a great challenge for clinicians due to inappropriate use of antibiotics. Antibiotics once considered being magic bullet is no more

\section{Keywords}

Antibiotics,

Treatment,

Surveillance,

Escherichia coli,

Resistance,

Meropenem,

Sensitivity

\section{Article Info}

Accepted:

22 July 2020

Available Online:

10 August 2020 having that title. Recommendation in AMR situation in India by Department of Biotechnology (DBT) and Treatment Guidelines for Antimicrobial Use by Indian Council of Medical Research (ICMR) India emphasized the need for regional surveillance starting from the level of state to an individual hospital has been motivation behind the study. Three hospitals in region of Cuddalore in Tamilnadu (India) have been the source of specimen for isolation of Escherichia coli during the period of January to July 2019. Antibiogram reports were collected during the study period and utilized for the study. Among 124 isolates Urine, High vaginal swab, Pus, Serum, Oral swab and Amniotic fluid occupied $81.45 \%$ (n-101), $08.06 \%(n-10), 5.64 \%(n-7), 2.41 \%(n-3), 1.62 \%(n-2)$ and $0.81 \%$ (n-1) respectively. Antibiogram for the Escherichia coli isolates revealed $90 \%$ of resistance to Cefepime, $68 \%$ to Ciprofloxacin, $50 \%$ to Tetracyclin, $30 \%$ to Meropenem etc. Similarly high percentage of sensitiveness was evident in Cefotaxime (95.94\%), Imipenum (95.91\%), Piperacillin/Tazobactem (86\%), Meropenem (70\%) etc. On comparison with the 2014 ICMR (Antimicrobial Susceptibility) data percentage of resistance and susceptibility has a drastic change both in resistance and sensitivity. From the study it is clear that the generic recommendations on antibiotic use at national level can't be a desirable solution or recommendations to clinicians. The study signifies the importance of local surveillance and infection control programs.

\section{Introduction}

E.coli is a gram negative, facultative anaerobic, rod shaped and coliform bacteria responsible for wound infection, food poisoning, lower urinary tract infection, upper urinary tract infection, septicemia, peritonitis, mastitis and pneumonia. In hospital community acquired infections UTI plays significant role due to use of catheters and administration of immunosuppressive drugs in extended hospitalization in extreme clinical 
conditions (Lim et al, 2017) (1). All the studies related to $\mathrm{E}$. coli have consistently confirmed its dominance in urine samples than other specimens.

Urinary tract infection (UTI) affects part of the urinary tract. If it affects the lower urinary tract it is named as a bladder infection (cystitis) and upper urinary tract it is known as kidney infection(pyelonephritis) (Flores et al, 2015) (2). The most predominant causative agent of infection is Escherichia coli, although the other bacteria may rarely be responsible. Female anatomy, sexual intercourse, diabetes, obesity, and family history are the responsible risk factors. UTIs are treated with a short course of antibiotics such as nitrofurantoin or trimethoprim/ sulfamethoxazole (Salvatore et al, 2011) (3).

In the last two decades, it is challenging to treat UTI infections caused by bacterial pathogens particularly that are transmitted in hospitals, because they are becoming resistant faster than we could develop new antibiotics. At present we have to depend on using toxic medications with potential and serious side effects due to the limitation that they are the only options available to treat infections from the multi-drug resistant bacteria (Wainwright and Milton 1989) (4).Lack of effective antibiotics is as serious as threat to security that will end in more mortality and sudden disease outbreak. Quick emergence of resistant bacteria/infections has again become a threat.

There are evidences in a study by Kalapana et $a l$, (2001) (5) in United States of America that the resistance by Escherichia coli to trimethoprim-sulfamethoxazole differed from region to region with a difference of $12 \%$ from $22 \%$ in western region to $10 \%$ in northeast region. In this study we have limited our surveillance within the region of Cuddalore-Tamilnadu (India) for knowing the resistance pattern of $E$. coli from different clinical specimens and comparing with ICMR guidelines as a point of reference.

\section{Materials and Methods}

Two hundred clinical samples like Urine, High vaginal swab, Pus, Serum, Oral swab and Amniotic fluid were collected in three private hospitals in Cuddalore and were streaked in selective media EMB agar (HiMedia) for isolation and identification of E.coli. Green Metallic sheen colonies were further confirmed for Gram staining, Motility and Standard Biochemical tests like, IMViC, CHO fermentation test, TSI test, Citrate utilization test, Catalase, Oxidase and Coagulase test. After the confirmation the confirmed isolates were inoculated into nutrient broth (Hi-Media) for multiplication. 0.5 MacFarl and standard was ensured for antibiotic sensitivity testing of all the 124 isolates to have $1.5 \times 10^{8}$ cells $/ \mathrm{ml}$ (Baker et al, 1983) (6).

After multiplying process standardized inoculums of each isolate were inoculated on to Mueller-Hinton antibiotic sensitivity medium (Hi-Media). Finally, all the isolates were tested for these under listed drug discs: Amikacin, Cefepime, Cefotaxime, Ceftazidime, Ciprofloxacin, Gentamycin, Imipenem, Meropenem, Piperacillin/ Tazobactem and Tetracycline. Standard antibiotic discs (Hi Media) were placed into the bacteria inoculated medium. Sterile conditions were taken care during this process. Incubation; waiting period was for 24 hours.

Depending upon the size of the zone, on comparison with the standard chart, and reviewing with guidelines of Clinical Laboratories Standard Institute (CLSI) for Antimicrobial Susceptibility Testing; the isolates were determined whether they are 
susceptible, moderately sensitive or resistant. The susceptibility breakpoints for each antibiotic were defined according to Clinical Laboratory Standards Institute (CLSI) guidelines (CLSI, 2011) (7). The susceptibility patterns were recorded under three categories like sensitive, intermediately sensitive/resistant and resistance. Final reports were collected and utilized for the study.

\section{Results and Discussion}

From the 200 clinical specimens collected 124 E. coli isolates were obtained with more no of isolates from urine sample having high frequency of $81.45 \% \quad(\mathrm{n}-101)$. It was followed by high vaginal swab, pus, serum, oral swab and amniotic fluid which are explained in Table 1.

According to Kibret and Abra (2011)(8) highest isolation percentage for $E$. coli was obtained in urine samples 203 (45.5\%). In a study by Devanand and Saxena, 2013 (9), among the gram negative isolates from urine sample $61.45 \%$ were E.coli, out of which $54.90 \%$ were isolated from inpatients of hospitals and $45.10 \%$ were from the community.

Our study results are in accordance with previous studies with the prevalence of $81.45 \%$ clearly indicating the dominance of E. coli in urinary tract infections. Ideal results have been reported by Poonam (2012) (10) also. Similarly many studies have reported the prevalence of UTI infections in Women than men indicating the high risk situation for the female inpatients.

In a study by Kibret and Abra in 2011(11) high resistance erythromycin (89.4\%), amoxicillin (86.0\%) and tetracycline (72.6\%) were recorded. Multi drug resistance of $74.6 \%$ and increased resistance rates to all antibiotics except ciprofloxacin were also recorded. E. coli isolates showed high rate of resistance to erythromycin, amoxicillin and tetracycline. Wani et al, (2009) (12) conducted a susceptibility study in E. coli clinical isolates and reported higher percentage of resistance to ceftazidime (99.2\%), cefotaxime $(99.2 \%)$ and ceftriaxone (99.5\%). Similarly, Rafay et al, (2007) (13) the study in tertiary care hospital in KashmirINDIA during $1^{\text {st }}$ August 2005 to $31^{\text {st }}$ July 2007, demonstrated $100 \%$ resistance of $E$. coli to cephalosporins. Duttaroy and Mehta (2005) (14) the study was done in Medical College Baroda-Gujarath in 2005, reported resistance of $E$. coli up to $75 \%$ to cefotaxime, $85 \%$ to ceftazidime and $60 \%$ to ceftriaxone. Kibret and Abera (2016) (15) the study was aimed at determining ESBL and antibiogram in Enterobacteriaceae isolates from clinical and drinking water sources in Bahir Dar City, Northwest Ethiopia during September 2013 to March 2015.

The antibiogram study of erythromycin, amoxicillin and tetracycline on E. coli isolated from UTI patients, and found high rates of resistance to erythromycin $(89.4 \%)$, amoxicillin (86.0\%) and tetracycline (72.6\%), respectively.

Complete susceptibility pattern of all E.coli isolates from Cuddalore region is found in the Table 2. Resistance to third generation cephalosporins cefepime, cefotaxime and ceftazidine is $90 \%, 04.05 \%$ and $04.00 \%$ respectively by our isolates. Resistance to cefotaxime and ceftazidime has been significantly reduced with a reduced difference of $75 \%$ by the isolates of Cuddalore region but with increased level of resistance against cefepime with $11 \%$ only. The samples chosen for framing ICMR Treatment Guidelines for Antimicrobial Use by ICMR India in 2017 have been hospitals of national significance with more inpatients of extended hospitalization (Jena et al, 2013) (16). 
Table.1 Showing prevalence of $E$. coli in clinical specimens

\begin{tabular}{|r|l|c|c|}
\hline S. No. & Name of the Specimen & No of Isolates $(\boldsymbol{E}$. coli) & Percentage of Prevalence (\%) \\
\hline $\mathbf{0 1}$ & Urine & 101 & 81.45 \\
\hline $\mathbf{0 2}$ & High Vaginal Swab & 10 & 08.06 \\
\hline $\mathbf{0 3}$ & Puss & 07 & 05.64 \\
\hline $\mathbf{0 4}$ & Serum & 03 & 02.41 \\
\hline $\mathbf{0 5}$ & Oral Swab & 02 & 01.62 \\
\hline $\mathbf{0 6}$ & Amniotic Fluid & 01 & 00.81 \\
\hline
\end{tabular}

Table.2 Showing the susceptibility pattern of the $E$. coli isolates in reference to susceptibility pattern described in ICMR Treatment Guidelines for Antimicrobial

Use by ICMR India in 2017(17)

\begin{tabular}{|c|c|c|c|c|c|c|c|c|}
\hline \multirow[t]{3}{*}{ S.No } & \multirow[t]{3}{*}{ Antibiotic } & \multicolumn{6}{|c|}{ E. coli isolates of our study (n-124) } & \multirow{3}{*}{$\begin{array}{l}\text { ICMR India } \\
\text { National \% of } \\
\text { Resistance }\end{array}$} \\
\hline & & \multicolumn{2}{|c|}{ Sensitive } & \multicolumn{2}{|c|}{ Intermed } & \multicolumn{2}{|c|}{ Resistant } & \\
\hline & & $\mathbf{N}$ & $\%$ & $\mathbf{n}$ & $\%$ & $\mathbf{n}$ & $\%$ & \\
\hline \multicolumn{9}{|c|}{ Third and fourth generation Cephalosporins } \\
\hline 01 & Cefepime & 12 & 10.00 & 0 & 0 & 112 & 90.00 & 79.00 \\
\hline 02 & Cefotaxime & 119 & 95.40 & 0 & 0 & 5 & 04.05 & 80.00 \\
\hline 03 & Ceftazidime & 18 & 14.66 & 101 & 81.33 & 5 & 04.00 & 81.00 \\
\hline \multicolumn{9}{|c|}{ Fluoroquinolones } \\
\hline 04 & Ciprofloxacine & 34 & 27.27 & 6 & 04.50 & 84 & 68.18 & 81.00 \\
\hline \multicolumn{9}{|c|}{ Aminoglycosides } \\
\hline 05 & Amikacin & 78 & 63.01 & 46 & 37.00 & o & 0 & 24.00 \\
\hline 06 & Gentamicin & 72 & 58 & 5 & 04.00 & 47 & 38.00 & 46.00 \\
\hline \multicolumn{9}{|c|}{ Carbapenems } \\
\hline 07 & Imipenem & 119 & 95.91 & 0 & 0 & 5 & 04.09 & 18.00 \\
\hline 08 & Meropenem & 87 & 70.10 & 0 & 0 & 37 & 30.00 & 35.00 \\
\hline \multicolumn{9}{|c|}{ Tetracyclines } \\
\hline 09 & Tetracycline & 62 & 50.00 & 0 & 0 & 62 & 50.00 & 64.00 \\
\hline \multicolumn{9}{|c|}{ Pecillin } \\
\hline 10 & $\begin{array}{l}\text { Piperacillin/Tazobact } \\
\text { um }\end{array}$ & 107 & 86.00 & 5 & 04.00 & 12 & 10.00 & 43.00 \\
\hline
\end{tabular}

The possibility for extensive consumption of life saving antibiotics would have surely led to increased exposure of pathogen (E.coli) to the antibiotics leading to prevalence of multidrug resistant bacteria in those areas. Empirical treatment based on guidelines as prophylactic measures might be also one of the reason for increased exposure. Another remarkable change that isolates have been more resistant to fourth generation cephalosporins than third generation on head to head study indicating the acquired resistance from extensive usage of fourth generation cephalosporins.

In a study by Tanzina et al., 2016 (18) in Bangladesh where antibiotic sensitivity test on E. coli isolates demonstrated that they 
were highly sensitive to Amikacin, Gentamycin, Netilmycin, Imipenem, Meropenem, Pipracillin-Tazobactam, Tobramycin, Nitrofurantoin, Azithromycin, Levofloxacin, and Ciprofloxacin.

In our study apart from third generation cephalosporin other group of antibiotics like fluoroquinolones, aminoglycosides, carbapenems, tetracyclines, penicillin have steadily shown drift in reduction of resistance percentage raging from minimum of $05 \%$ under carbapenems to maximum of $33 \%$ in penicillin group (Piperacillin/Tazobactem), a positive sign to revert back to conventional antibiotics until the sensitivity reaches $100 \%$ for the above classes (Mandal et al., 2001) (19). May be the drift in the form of adaptive resistance by E.coli to newer generation, costly, life saving and high side effect antibiotics will lead to change in efflux mechanisms and receptor modulations.

In conclusion to develop a nationwide policy in 2017 the susceptibility pattern data of 2014 has been utilized clearly spacing 2015 and 2016. "AMR situation in India by DBT" Govt. of India (20) has searched the PubMed and Google Scholar databases relating to AMR in India, using following terms: "antimicrobial OR antibiotic and resistance and India." The search was limited to the last five years (July 1, 2012, to June 30, 2017) (Sumanth et al, 2017) (21). Comparatively it would give a comprehensive picture on nationwide susceptibility pattern but limited to the zones/regions exposed to research centers or institutes only.

The study clearly emphasizes local antimicrobial surveillance from hospital level to combat the major threat (antimicrobial resistance) to mankind. Only by knowing the extent of resistance and sensitivity can appropriate choices of antibiotic can be made.

\section{Declaration of interest}

The authors report no conflict of interest. The authors alone are responsible for the content and writing of this article.

\section{Acknowledgement}

No funding was obtained for this study.

Ethical approval is not required for this study.

\section{References}

1. Lim, J., Yoon, J.W. and Hovde, C.J. (2017). A Brief Overview of Escherichia coli O157:H7 and Its Plasmid O157. J.Microbiol Biotechnol. 20 (1): pp05-14.

2. Flores-Mireles, A.L., Walker, J.N., Caparon, M. and Hultgren, S.J. (2015). Urinary tract infections: epidemiology, mechanisms of infection and treatment options. Nature Reviews. Microbiology. 13 (5): pp269-84.

3. Salvatore, S., Cattoni, E., Siesto, G., Serati, M., Sorice, P. and Torella, M. (2011). Urinary tract infections in women. European journal of obstetrics, gynecology, and reproductive biology. 156 (2): pp131-136.

4. Wainwright and Milton (1989). Moulds in ancient and more recent medicine Mycologist. Vol.3 (1), pp.21-23.

5. Kalpana, G., Daniel, F. S., David, M. and Walter, E. S. (2001). Antimicrobial Resistance among Uropathogens that Cause Community-Acquired Urinary Tract Infections in Women: A Nationwide Analysis. Clinical Infectious Diseases. 33(1): pp89-94.

6. Baker, C.N., Thornsberry, C. and Hawkinson, R.W. (1983). Inoculum standardization in antimicrobial susceptibility tests: evaluation of the overnight agar cultures and the rapid 
inoculum standardization system. $J$ Clin Micro, 17: pp450-457.

7. Clinical and Laboratory Standards Institute (CLSI). 2012. Available: clsi.org/standards. Accessed: 25 December 2011.

8. 11, 15. Kibret, M. and Abera, B. (2011). Antimicrobial susceptibility patterns of E. coli from clinical sources in northeast Ethiopia. Afri Health Sciences, 11(1): pp 40-45.

9. Devanand, P. and Saxena, R.S. (2013). Prevalence and antimicrobial susceptibility pattern of Escherichia coli in hospital acquired and community acquired patients related to urinary tract infection in India.Journal of Applied Pharmaceutical Science, 3 (08): pp. 124-132.

10. Poonam, V. (2012) A study on isolation of E.coli bacteria from different clinical specimens in Raipur. Indian Streams Research Journal. 2(9): pp. 01-04.

12. Wani, K.A., Thakur, M.A., Siraj, F.A., Fomdia, B. and Gulnaz, B. (2009), extended spectrum B-lactamase mediated resistance in Escherichia coli in a tertiary care hospital. Int $J$ Health Sci (Qassim) 3: pp. 155-163.

13. Rafay, A.M., Al-Muharmi, Z., Toki, R. (2007). Prevalence of extendedspectrum beta-lactamases-producing isolates over a 1-year period at a University Hospital in Oman. Saudi Med J 28: pp. 22-27.

14. Duttaroy, B. and Mehta. S. (2005) Extended spectrum b lactamases (ESBL) in clinical isolates of Klebsiella pneumoniae and Escherichia coli. Indian J Pathol Microbiol 48: pp. 45-48.

16. Jena. J., Debata, N.K. and Subudhi, E. (2013) Prevalence of extendedspectrum-beta-lactamase and metallobeta-lactamase producing multi drug resistance gram- negative bacteria from urinary isolates. Indian J Med Microbiol 31: pp.420-421.

17. Indian Council of Medical Research ICMR Antimicrobial Use Guidelines 2017

18. Tanzina, A., Mohammad, J.H., Md Sumon, K., Hoomyra, S., Kaniz, F., Sohana, A.S. and Suvamoy, D. (2016). Isolation, identification and antimicrobial susceptibility pattern analysis of Escherichia coli isolated from clinical samples of Bangladesh. Asian Journal of Biomedical and Pharmaceutical Sciences, 6(54): pp.1316.

19. Mandal, P., Kapil, A., Goswami, K., Das, B. and Dwivedi, S.N. (2001) UropathogenicEscherichia coli causing urinary tract infections. Indian $\mathrm{J}$ Med Res. 114: pp. 207-11.

20.Scoping Report on Antimicrobial Resistance in India by Department of biotechnology November 2017.

21. Sumanth, G., Jyoti, J., Anna, T., Anjana, S.L. and Ramanan, L. (2017). Scoping Report on Antimicrobial Resistance in India. Washington, DC: Center for Disease Dynamics, Economics \& Policy.

\section{How to cite this article:}

Sathish, V. D., Guru Prasad Mohanta, Elizabeth Anna Mathew and Renga Ramanujam, J. 2020. Dynamism in Resistance Pattern of Escherichia coli -A Drift from Indian Council of Medical Research (ICMR)-Antimicrobial Use Guidelines. Int.J.Curr.Microbiol.App.Sci. 9(08): 29112916. doi: https://doi.org/10.20546/ijcmas.2020.908.325 